\title{
ARITHMETIC CALCULUS OF FOURIER TRANSFORMS BY IGUSA LOCAL ZETA FUNCTIONS
}

\author{
TATSUO KIMURA
}

\begin{abstract}
We show the possibility of explicit calculation of the Fourier transforms of complex powers of relative invariants of some prehomogeneous vector spaces over $\mathbb{R}$ by using the explicit form of $p$-adic Igusa local zeta functions.
\end{abstract}

Let $(G, \rho, V)$ be a regular prehomogeneous vector space defined over $\mathbb{R}$, and $f_{1}, \ldots, f_{r}$ the basic $\mathbb{R}$-relative invariants of $(G, \rho, V)$ (cf. [F. Sato 2, p. 444]). For explicit calculation of Fourier transforms of the complex power

$$
|f(x)|^{s}=\prod_{i=1}^{r}\left|f_{i}(x)\right|^{s_{i}}
$$

with $s=\left(s_{1}, \ldots, s_{r}\right) \in \mathbb{C}^{r}$, two general methods are known. When $(G, \rho, V)$ has finitely many $G$-orbits, one can use a microlocal calculus established by $\mathbf{M}$. Kashiwara according to M. Sato's idea (see p. 404 in [M], [K-K-M]). When $r=1$ and the relative invariant is linear for each variable, one can use Igusa's method (see p. 8 in [Igusa 2]).

In this paper, we shall show the possibility of calculation for some cases by using the explicit form of the Igusa's local zeta function, based on the idea of Iwasawa-Tate theory [Iwasawa], [Tate].

As an example, we shall calculate the Fourier transform of $|f(x)|^{s}$ for $\left(G L_{1}^{4} \times\right.$ $\left.S L_{2 m+1}, \Lambda_{2} \oplus \Lambda_{1} \oplus \Lambda_{1}^{*} \oplus \Lambda_{1}^{*}, \mathrm{Alt}_{2 m+1} \oplus \mathrm{Aff}^{2 m+1} \oplus \mathrm{Aff}^{2 m+1} \oplus \mathrm{Aff}^{2 m+1}\right)$ which has infinitely many orbits and $r=4$ so that it has not been calculated by other methods.

The Igusa local zeta function of this prehomogeneous vector space has been explicitly calculated by [Hosokawa]. Our method is applicable for a prehomogeneous vector space with $Z_{a}=\tau Z_{m}$ for some constant $\tau>0$. For example, irreducible (resp. simple, 2-simple of type I) regular prehomogeneous vector spaces with finitely many adelic open orbits satisfy $Z_{a}=\tau Z_{m}$ (see [Igusa 4] and $[\mathrm{K}-\mathrm{K}])$.

This paper has four sections: $\S 1$, Preliminaries; $\S 2$, Functional equation; $\S 3$, Unramified $\Gamma$-factors and Igusa local zeta functions; $\S 4$, Arithmetic calculus (example). The last section of this paper was completed when the author was

Received by the editors July 27, 1993 and, in revised form, March 30, 1994.

1991 Mathematics Subject Classification. Primary 32M12, 20G20; Secondary 11 S40.

Key words and phrases. Prehomogeneous vector spaces, Iwasawa-Tate theory. 
staying at the Japan-U.S. Mathematics Institute (JAMI), 1993, at The Johns Hopkins University. The author would like to express his hearty thanks to Professor J. Igusa and Professor F. Sato whose encouragement and suggestions stimulated this work.

\section{Preliminaries}

Let $\tilde{G}$ be a connected linear algebraic group and $\rho: \tilde{G} \rightarrow G L(V)$ a rational representation of $\tilde{G}$ on a finite-dimensional vector space $V$. Put $G=\rho(\tilde{G}) \subset$ $G L(V)$. When $V$ has a Zariski-dense $G$-orbit $Y$, we call a triplet $(\tilde{G}, \rho, V)$ (or a pair $(G, V)$ ) a prehomogeneous vector space (abbrev. P.V.). A point of $Y$ is called a generic point and the isotropy subgroup at a generic point is called a generic isotropy subgroup which is unique up to isomorphisms. The complement $S$ of $Y$ is a Zariski-closed set which is called the singular set of $(G, V)$. An irreducible component $S_{i}$ of codimension one is the zeros of some irreducible polynomial $f_{i}(x) \quad(i=1, \ldots, r)$. Then these polynomials are algebraically independent relative invariants, i.e., $f_{i}(\rho(g) x)=\chi_{i}(g) f_{i}(x)$ for $g \in \tilde{G}$ and $x \in V$ with some rational characters $\chi_{i}$ of $\tilde{G}$. Moreover any relatively invariant rational function $f(x)$ is of the form $f(x)=c f_{1}(x)^{m_{1}} \cdots f_{r}(x)^{m_{r}}$ with $\left(m_{1}, \ldots, m_{r}\right) \in \mathbb{Z}^{r}$ and some constant $c$ (see p. 60 in [S-K]). We call $f_{1}, \ldots, f_{r}$ the basic relative invariants of $(\tilde{G}, \rho, V)$. Now a P.V. is called regular if the Hessian $H_{f}(x)=\operatorname{det}\left(\frac{\partial^{2} f}{\partial x_{i} \partial x_{j}}(x)\right)$ is not identically zero for some relative invariant $f(x)$. In this case, we have $\operatorname{det} \rho(g)^{2}=\chi_{1}(g)^{2 \kappa_{1}} \cdots \chi_{r}(g)^{2 \kappa_{r}}$ for some $\kappa=\left(\kappa_{1}, \ldots, \kappa_{r}\right) \in\left(\frac{1}{2} \mathbb{Z}\right)^{r}$ (see p. 61 in [S-K]). When $r=1$, we have $\kappa=\frac{n}{d}$ with $d=\operatorname{deg} f$ and $n=\operatorname{dim} V$.

When $G$ is reductive, it is regular if and only if a generic isotropy subgroup is reductive, and it is so if and only if the singular set $S$ is a hypersurface (see p. 73 in $[\mathrm{S}-\mathrm{K}])$. In this case, without essential loss of generality, we may assume that a generic isotropy subgroup is semisimple (cf. $\S 3$ and $\S 4$ in $[\mathrm{K}-\mathrm{K}])$. Let $k$ be an algebraic number field. From now on, we assume that $(G, V)$ is a reductive P.V. defined over $k$ with a connected semisimple generic isotropy subgroup, and all coefficients of $f_{i}(x)$ are in $k$. We denote by $G_{A}, V_{A}$, etc., the adelization of $G, V$, etc., with respect to $k$. Let $\Omega\left(k_{A}^{\times} / k^{\times}\right)$be the space of quasicharacters of the idele class group $k_{A}^{\times} / k^{\times}$of $k$ and $\mathfrak{S}\left(V_{A}\right)$ the SchwarzBruhat space of $V_{A}$. For $\omega=\left(\omega_{1}, \ldots, \omega_{r}\right) \in \Omega\left(k_{A}^{\times} / k^{\times}\right)^{r}$, we write $\omega(\chi(g))=$ $\omega_{1}\left(\chi_{1}(g)\right) \cdots \omega_{r}\left(\chi_{r}(g)\right)$ and $\omega(f(x))=\omega_{1}\left(f_{1}(x)\right) \cdots \omega_{r}\left(f_{r}(x)\right)\left(g \in G_{A}, x \in\right.$ $\left.Y_{A}\right)$ for simplicity. Now we define the two adelic zeta-functions $Z_{a}(\omega, \Phi)$ and $Z_{m}(\omega, \Phi)$ of $(G, V)$.

$$
\begin{gathered}
Z_{a}(\omega, \Phi)=\int_{G_{A} / G_{k}} \omega(\chi(g)) \sum_{\xi \in Y_{k}} \Phi(g \xi) d_{G_{A}}(g), \\
Z_{m}(\omega, \Phi)=\int_{Y_{A}} \omega(f(x)) \Phi(x) d_{Y_{A}}(x) \quad\left(\Phi \in \mathfrak{S}\left(V_{A}\right)\right) .
\end{gathered}
$$


Here $d_{G_{A}}$ is a right-invariant measure on $G_{A}$ while $d_{Y_{A}}$ is a $G_{A}$-invariant measure on $Y_{A}$. Since a generic isotropy subgroup is connected semisimple, we may take the same convergence factor for $d_{G_{A}}$ and $d_{Y_{A}}$. Note that, for the simplest P.V.( $\left.G L_{1}, \mathrm{Aff}^{1}\right)$, we have

$$
Z_{m}=\int_{k_{A}^{\times}} \omega(x) \Phi(x) d^{\times} x=\int_{k_{A}^{\times} / k^{\times}} \omega(x) \sum_{\xi \in k^{\times}} \Phi(x \xi) d^{\times} x=Z_{a}
$$

which appears in the original Iwasawa-Tate theory. For $s=\left(s_{1}, \ldots, s_{r}\right) \in \mathbb{C}^{r}$ and $x=\left(x_{1}, \ldots, x_{r}\right) \in k_{A}^{\times^{r}}$, we put $\omega_{s}(x)=\left|x_{1}\right|_{A}^{s_{1}} \cdots\left|x_{r}\right|_{A}^{s_{r}}$. For any $\omega \in$ $\Omega\left(k_{A}^{\times} / k^{\times}\right)^{r}$, we have $|\omega(x)|=\omega_{\sigma}(x)$ for some $\sigma=\left(\sigma_{1}, \ldots, \sigma_{r}\right) \in \mathbb{R}^{r}$. In this case, we write $\sigma(\omega)=\left(\sigma_{1}, \ldots, \sigma_{r}\right) \in \mathbb{R}^{r}$. It is known that $Z_{m}(\omega, \Phi)$ is absolutely convergent when $\sigma(\omega)=\left(\sigma_{1}, \ldots, \sigma_{r}\right)>\kappa=\left(\kappa_{1}, \ldots, \kappa_{r}\right)$, i.e., $\sigma_{i}>\kappa_{i}$ for $i=1, \ldots, r$ (see [Ono], p. 90 in [F. Sato 1]). One can see that $Z_{m}(\omega, \Phi)$ has an Euler product $Z_{m}(\omega, \Phi)=\prod_{v \in \Sigma} Z_{v}\left(\omega_{v}, \Phi_{v}\right)$ for $\Phi=$ $\bigotimes_{v \in \Sigma} \Phi_{v}$ where $\Sigma$ denotes the set of places of $k$. We can express the local factor $Z_{v}\left(\omega_{v}, \Phi_{v}\right)$ by the Igusa local zeta function for almost all finite places $v$. We define $Z_{v}(s)$ to be the Igusa local zeta function $\int_{O_{v}^{n}}\left|f_{1}(x)\right|^{s_{1}} \cdots\left|f_{r}(x)\right|^{s_{r}} d x$ with $\int_{O_{v}^{n}} d x=1$. It is a rational function of $t_{i}=q_{v}^{-s_{i}}(i=1, \ldots, r)$.

Theorem 1.1 ([Igusa 4], [K-K]). Let $(G, \rho, V)$ be an irreducible (resp. simple, 2-simple of type I) P.V. defined and split over an algebraic number field $k$ satisfying $\left|G_{A} \backslash Y_{A}\right|<+\infty$. Then we have $Z_{a}(\omega, \Phi)=\tau Z_{m}(\omega, \Phi)$ for some constant $\tau>0$.

From now on, we assume that $Z_{a}=\tau Z_{m}$ for some $\tau>0$ so that $Z_{a}(\omega, \Phi)$ converges absolutely when $\sigma(\omega)>\kappa$.

\section{Functional EQUATION}

We have assumed that $(\tilde{G}, \rho, V)$ or $(G, V)$ is a reductive regular P.V. defined and split over an algebraic number field with a connected semisimple generic isotropy subgroup and $Z_{a}(\omega, \Phi)=\tau Z_{m}(\omega, \Phi)$ for some $\tau>0$. We may assume that $f_{i}(x) \in O_{k}[x](i=1, \ldots, r)$ where $O_{k}$ is the maximal order of $k$. Let $\rho^{*}$ be the contragredient representation of $\rho$ on the dual vector space $V^{*}$ of $V$. Then $\left(\tilde{G}, \rho^{*}, V^{*}\right)$ is also a reductive regular P.V. defined over $k$ with the singular set $S^{*}$, and $Y^{*}=V^{*}-S^{*}$. Since $\tilde{G}$ is reductive, we have the basic relative invariants $f_{1}^{*}, \ldots, f_{r}^{*}$ satisfying

$$
f_{i}^{*}\left(\rho^{*}(g) y\right)=\chi_{i}(g)^{-1} f_{i}^{*}(y) \quad(i=1, \ldots, r)
$$

for $g \in \tilde{G}$ and $y \in V^{*}$. By taking a basis, compatible with the $k$-structure of $(G, \rho, V)$, we may identify $V$ and $V^{*}$ with Aff ${ }^{n}$ so that $\rho^{*}(\tilde{g})={ }^{t} \rho(\tilde{g})^{-1}$ for $\tilde{g} \in \tilde{G}$. We write $g^{*} \cdot y={ }^{t} g^{-1} y$ for $g=\rho(\tilde{g}) \in G=\rho(\tilde{G})$, and $y \in V^{*}$. We define $Z_{a}^{*}(\omega, \Psi)$ and $Z_{m}^{*}(\omega, \Psi)$ as follows:

$$
Z_{a}^{*}(\omega, \Psi)=\int_{G_{A} / G_{k}} \omega\left(\chi^{-1}(g)\right) \sum_{\eta \in Y_{k}^{*}} \Psi\left(g^{*} \eta\right) d_{G_{A}}(g),
$$




$$
Z_{m}^{*}(\omega, \Psi)=\int_{Y_{A}^{*}} \omega\left(f^{*}(y)\right) \Psi(y) d_{Y_{A}^{*}}(y) \quad\left(\Psi \in \mathfrak{S}\left(V_{A}^{*}\right)\right) .
$$

For any place $v \in \Sigma$ of $k$, let $k_{v}$ be the local field corresponding to $v$. For $\Phi_{v} \in \mathfrak{S}\left(V_{v}\right)$ with $V_{v}=k_{v}^{n}$, let $\hat{\Phi}_{v}$ be its Fourier transform with respect to the self-dual measure so that $\hat{\boldsymbol{\Phi}}_{v}(x)=\Phi_{v}(-x)$ holds. For a finite place $v$, the self-dual measure $d_{1} x$ satisfies $\int_{O_{v}^{n}} d_{1} x=N\left(d_{v}\right)^{-\frac{n}{2}}$ where $d_{v}$ is the different of $k_{v}$, and hence note that it is not the measure $d x$ satisfying $\int_{O_{v}^{n}} d x=1$ which appears in the definition of the Igusa local zeta function. For $\omega_{v}=$ $\left(\omega_{v}^{1}, \ldots, \omega_{v}^{r}\right)$ where $\omega_{v}^{i}$ is a quasicharacter of $k_{v}^{\times}$, we define the local zeta function $Z_{v}$ by

$$
Z_{v}\left(\omega_{v}, \Phi_{v}\right) \stackrel{\text { def }}{=} \int_{Y_{v}} \omega_{v}(f(x)) \Phi_{v}(x) d_{Y_{v}}(x) .
$$

Similarly we define $Z_{v}^{*}$ by

$$
Z_{v}^{*}\left(\omega_{v}, \Psi_{v}\right) \stackrel{\text { def }}{=} \int_{Y_{v}^{*}} \omega_{v}\left(f^{*}(y)\right) \Psi_{v}(y) d_{Y_{v}^{*}}(y) .
$$

Lemma 2.1. For any infinite place $v \in \Sigma_{\infty}$, there exists $\Phi_{v} \in \mathfrak{S}\left(V_{v}\right)$ satisfying $\boldsymbol{\Phi}_{v} \neq 0,\left.\boldsymbol{\Phi}_{v}\right|_{S_{v}}=0$, and $\left.\hat{\boldsymbol{\Phi}}_{v}\right|_{S_{v}^{*}}=0$.

Proof. Put $F^{*}=f_{1}^{*} \cdots f_{r}^{*}$ so that $S^{*}$ is the zeros of $F^{*}$. Take a nonzero $\Phi_{o} \in C_{0}^{\infty}\left(Y_{v}\right)$ and put $\Phi_{v}=F^{*}\left(\operatorname{grad}_{x}\right) \Phi_{0}$. Since $\hat{\Phi}_{v}(y)= \pm F^{*}(y) \hat{\Phi}_{0}(y)$, this $\Phi_{v}$ satisfies our conditions. This is a well-known argument.

We denote by $A_{f}$ the restricted direct product over the finite places.

Lemma 2.2. There exists $\Phi_{f} \in \mathfrak{S}\left(V_{A_{f}}\right)$ satisfying $\Phi_{f} \neq 0, \Phi_{f} \mid S_{A_{f}}=0$, and $\hat{\Phi}_{f} \mid s_{\boldsymbol{A}_{f}}=0$.

Proof. For a finite place $v$, take $a \in V\left(O_{v}\right)=O_{v}^{n}$ satisfying $|F(a)|_{v}=1$ with $F=f_{1} \cdots f_{r}$. Since $|F(a+\pi b)|_{v}=|F(a)+\pi c|_{v}=1$ for any $b \in O_{v}^{n}$, we have $a+\pi O_{v}^{n} \subset Y_{v}$. Let $\Phi_{v}$ be the characteristic function of $a+\pi O_{v}^{n}$. Then we have $\boldsymbol{\Phi}_{v} \in \mathfrak{S}\left(V_{v}\right), \Phi_{v} \neq 0$, and $\left.\Phi_{v}\right|_{S_{v}}=0$. Take a finite place $v^{\prime} \neq v$ and let $\Phi_{v^{\prime}}$ be the Fourier transform of the characteristic function of $a^{\prime}+\pi O_{v^{\prime}}^{n}$ with $\left|F^{*}\left(a^{\prime}\right)\right|_{v^{\prime}}=1$. Since $\Phi_{v^{\prime}} \neq 0$ and $\left.\hat{\Phi}_{v^{\prime}}\right|_{s_{v^{\prime}}}=0$, if we put $\Phi_{f}=\Phi_{v} \cdot \Phi_{v^{\prime}} \cdot \prod_{w \neq v, v^{\prime}} \operatorname{ch}_{O_{w}^{n}}$ where $\operatorname{ch}_{O_{w}^{n}}$ is the characteristic function of $O_{w}^{n}$, this satisfies our condition.

To prove the following proposition, we shall use the argument similar to the one on p. 468 in [F. Sato 2].

Proposition 2.3. For $\Phi \in \mathfrak{S}\left(V_{A}\right)$ satisfying $\left.\Phi\right|_{S_{A}}=\left.\hat{\Phi}\right|_{S_{A}^{*}}=0$, the functions $Z_{a}(\omega, \Phi)$ and $Z_{a}^{*}(\hat{\omega}, \hat{\Phi})$ can be analytically continued to the whole $\Omega\left(k_{A}^{\times} / k^{\times}\right)^{r}$ and they satisfy a functional equation $Z_{a}(\omega, \Phi)=Z_{a}^{*}(\hat{\omega}, \hat{\Phi})$ where $\hat{\omega}=$ $\omega_{\kappa} \omega^{-1}$ and $\hat{\boldsymbol{\Phi}}$ is the Fourier transform of $\boldsymbol{\Phi}$.

Proof. Note that $Z_{a}(\omega, \Phi)$ (resp. $\left.Z_{a}^{*}(\hat{\omega}, \hat{\Phi})\right)$ converges absolutely on $A=$ $\left\{\omega \in \Omega\left(k_{A}^{\times} / k^{\times}\right)^{r} ; \sigma(\omega)>\kappa\right\} \quad$ (resp. $A^{*}=\left\{\omega \in \Omega\left(k_{A}^{\times} / k^{\times}\right)^{r} ; \sigma(\omega)<0\right\}$ ). Take $\omega_{b} \in A$ and $\omega_{b^{*}} \in A^{*}$ with $b, b^{*} \in \mathbb{Z}^{r}$. For $c=b-b^{*}$ and $\varepsilon= \pm 1$, 
put

$$
Z_{a}^{\varepsilon}(\omega, \Phi)=\int_{\substack{G_{A} / G_{k} \\ \omega_{c}(\chi(g))^{\varepsilon} \geq 1}} \omega(\chi(g)) \sum_{\xi \in Y_{k}} \Phi(g \xi) d_{G_{A}}(g)
$$

and

$$
Z_{a}^{* \varepsilon}(\hat{\omega}, \hat{\Phi})=\int_{\substack{G_{A} / G_{k} \\ \omega_{c}(\chi(g))^{\varepsilon} \geq 1}} \omega(\chi(g)) \omega_{\kappa}\left(\chi^{-1}(g)\right) \sum_{\eta \in Y_{k}^{*}} \hat{\Phi}\left(g^{*} \eta\right) d_{G_{A}}(g)
$$

We have $Z_{a}(\omega, \Phi)=Z_{a}^{+}(\omega, \Phi)+Z_{a}^{-}(\omega, \Phi)$ and $Z_{a}^{*}(\hat{\omega}, \hat{\Phi})=Z_{a}^{*+}(\hat{\omega}, \hat{\Phi})+$ $Z_{a}^{*-}(\hat{\omega}, \hat{\Phi})$. Then $Z_{a}^{\varepsilon}(\omega, \Phi)$ converges absolutely on

$$
B^{\varepsilon}=\left\{\omega \in \Omega\left(k_{A}^{\times} / k^{\times}\right)^{r} ; \omega \omega_{c}^{\varepsilon t} \in A \text { for some } t \geq 0\right\}
$$

and $Z_{a}^{* \varepsilon}(\hat{\omega}, \hat{\Phi})$ converges absolutely on

$$
B^{* \varepsilon}=\left\{\omega \in \Omega\left(k_{A}^{\times} / k^{\times}\right)^{r} ; \omega \omega_{c}^{\varepsilon t} \in A^{*} \text { for some } t \geq 0\right\} .
$$

Note that we have $B^{+}=B^{*-}=\Omega\left(k_{A}^{\times} / k^{\times}\right)^{r}$ since $\sigma\left(\omega_{c}\right)>0$. For example, for any $\omega \in \Omega\left(k_{A}^{\times} / k^{\times}\right)^{r}$, take $t>0$ satisfying $\sigma\left(\omega \omega_{c}^{t}\right)>\kappa$, i.e., $\omega \omega_{c}^{t} \in A$. Then we have

$$
\begin{aligned}
\infty & >Z_{a}^{+}\left(\omega \omega_{c}^{t}, \Phi\right)=\int_{\substack{G_{A} / G_{k} \\
\omega_{c}(\chi(g)) \geq 1}} \omega_{c}(\chi(g))^{t} \cdot \omega(\chi(g)) \sum_{\xi \in Y_{k}} \Phi(g \xi) d_{G_{A}}(g) \\
& \geq Z_{a}^{+}(\omega, \Phi) .
\end{aligned}
$$

On $B^{\varepsilon} \cap B^{* \varepsilon}$, by the adelic Poisson summation formula

$$
\sum_{\xi \in Y_{k}} \Phi(g \xi)=\omega_{\kappa}\left(\chi^{-1}(g)\right) \sum_{\eta \in Y_{k}^{*}} \hat{\Phi}\left(g^{*} \eta\right)
$$

for our $\Phi$, we have $Z_{a}^{\varepsilon}(\omega, \Phi)=Z_{a}^{* \varepsilon}(\hat{\omega}, \hat{\Phi})$ so that $Z_{a}^{\varepsilon}(\omega, \Phi)$ and $Z_{a}^{* \varepsilon}(\hat{\omega}, \hat{\Phi})$ are analytically continued to $B^{\varepsilon} \cup B^{* \varepsilon}=\Omega\left(k_{A}^{\times} / k^{\times}\right)^{r}$. Hence $Z_{a}(\omega, \Phi)=$ $Z_{a}^{+}(\omega, \boldsymbol{\Phi})+Z_{a}^{-}(\omega, \boldsymbol{\Phi})$ and $Z_{a}^{*}(\hat{\omega}, \hat{\boldsymbol{\Phi}})=Z_{a}^{*+}(\hat{\boldsymbol{\omega}}, \hat{\boldsymbol{\Phi}})+Z_{a}^{*-}(\hat{\boldsymbol{\omega}}, \hat{\boldsymbol{\Phi}})$ are analytically continued to the whole $\Omega\left(k_{A}^{\times} / k^{\times}\right)^{r}$ and they satisfy the functional equation $Z_{a}(\omega, \Phi)=Z_{a}^{*}(\hat{\omega}, \hat{\Phi})$.

Theorem 2.4. For our P.V. $(G, V)$, the Euler product $\prod_{v} Z_{v}\left(\omega_{v}, \Phi_{v}\right)$ has a functional equation:

$$
\prod_{v \in \Sigma} Z_{v}\left(\omega_{v}, \Phi_{v}\right)=\prod_{v \in \Sigma} Z_{v}^{*}\left(\hat{\omega}_{v}, \hat{\Phi}\right)
$$

where $\hat{\omega}_{v}=\left(\omega_{v}\right)_{\kappa} \omega_{v}^{-1}$.

Proof. For any $\boldsymbol{\Phi}_{f} \in \mathfrak{S}\left(V_{A_{f}}\right)$, put $\boldsymbol{\Phi}=\Phi_{\infty}^{0} \otimes \Phi_{f}$ where $\Phi_{\infty}^{0}\left|s_{\infty}=\hat{\Phi}_{\infty}^{0}\right| s_{\infty}^{*}=0$ and $\Phi_{\infty}^{0} \neq 0$ (cf. Lemma 2.1). Then we have $\left.\Phi\right|_{S_{A}}=\left.\hat{\Phi}\right|_{S_{A}^{*}}=0$ and by 
Proposition 2.3, we obtain

$$
\begin{array}{r}
Z_{\infty}\left(\omega_{\infty}, \Phi_{\infty}^{0}\right) \cdot Z_{f}\left(\omega_{f}, \Phi_{f}\right)=Z_{\infty}^{*}\left(\hat{\omega}_{\infty}, \hat{\Phi}_{\infty}^{0}\right) \cdot Z_{f}^{*}\left(\hat{\omega}_{f}, \hat{\Phi}_{f}\right) \\
\quad \text { for any } \Phi_{f} .
\end{array}
$$

On the other hand, for any $\Phi_{\infty} \in \mathfrak{S}\left(V_{\infty}\right)$, put $\Phi=\Phi_{\infty} \otimes \Phi_{f}^{0}$ with $\Phi_{f}^{0}$ as in Lemma 2.2. Similarly we have

$$
Z_{\infty}\left(\omega_{\infty}, \Phi_{\infty}\right) \cdot Z_{f}\left(\omega_{f}, \Phi_{f}^{0}\right)=Z_{\infty}^{*}\left(\hat{\omega}_{\infty}, \hat{\Phi}_{\infty}\right) \cdot Z_{f}^{*}\left(\hat{\omega}_{f}, \hat{\Phi}_{f}^{0}\right)
$$

Multiplying equations (2.4) and (2.5), we have $Z_{m}(\omega, \Phi) Z_{m}\left(\omega, \Phi^{0}\right)=$ $Z_{m}^{*}(\hat{\omega}, \hat{\Phi}) Z_{m}^{*}\left(\hat{\omega}, \hat{\Phi}^{0}\right)$ with $\Phi=\Phi_{\infty} \otimes \Phi_{f}$ and $\Phi^{0}=\Phi_{\infty}^{0} \otimes \Phi_{f}^{0}$. By $(2.4)$ with $\Phi_{f}=\Phi_{f}^{0}$, we have $Z_{m}\left(\omega, \Phi^{0}\right)=Z_{m}^{*}\left(\hat{\omega}, \hat{\Phi}^{0}\right)$ and hence $Z_{m}(\omega, \Phi)=$ $Z_{m}^{*}(\hat{\omega}, \hat{\Phi})$ holds for any $\Phi$.

\section{UNRAMIFIED $\Gamma$-FACTORS AND IGUSA LOCAL ZETA FUNCTIONS}

By Theorem 2.4, we have

$$
\begin{aligned}
& Z_{v}\left(\omega_{v}, \boldsymbol{\Phi}_{v}\right) \cdot \prod_{w \neq v} Z_{m}\left(\omega_{w}, \Phi_{w}\right)=Z_{v}^{*}\left(\hat{\omega}_{v}, \hat{\Phi}_{v}\right) \cdot \prod_{w \neq v} Z_{m}^{*}\left(\hat{\omega}_{w}, \hat{\Phi}_{w}\right) \\
& Z_{v}^{*}\left(\hat{\omega}_{v}, \hat{\Psi}_{v}\right) \cdot \prod_{w \neq v} Z_{m}^{*}\left(\hat{\omega}_{w}, \hat{\boldsymbol{\Phi}}_{w}\right)=Z_{v}\left(\omega_{v}, \Psi_{v}\right) \cdot \prod_{w \neq v} Z_{m}\left(\omega_{w}, \Phi_{w}\right) .
\end{aligned}
$$

Multiplying (3.1) and (3.2), we have

$$
Z_{v}\left(\omega_{v}, \Phi_{v}\right) \cdot Z_{v}^{*}\left(\hat{\omega}_{v}, \hat{\Psi}_{v}\right)=Z_{v}^{*}\left(\hat{\omega}_{v}, \hat{\Phi}_{v}\right) \cdot Z_{v}\left(\omega_{v}, \Psi_{v}\right)
$$

Namely, there exists $\Gamma_{v}\left(\omega_{v}\right)$ satisfying

$$
Z_{v}\left(\omega_{v}, \Phi_{v}\right)=\Gamma_{v}\left(\omega_{v}\right) Z_{v}^{*}\left(\hat{\omega}_{v}, \hat{\Phi}_{v}\right) \text { for any } \Phi_{v} \in \mathfrak{S}\left(V_{v}\right) .
$$

Thus we can obtain the local functional equation. However, it has already been obtained in more general cases [Igusa 1], [Gyoja].

In this section, we express a $\Gamma$-factor $\Gamma_{v}\left(\omega_{v}\right)$ by the Igusa local zeta function $Z_{v}(s)$ when $\omega_{v}$ is an unramified quasicharacter $\omega_{v, s}=\left(\left.||\right|_{v} ^{s_{1}}, \ldots,\left.||\right|_{v} ^{s_{r}}\right)$ for a finite place $v$ of $k$. For one variable case $(r=1)$, including ramified case, see [Igusa 2].

Lemma 3.1. For a finite place $v$ of $k$, we have $Z_{v}\left(\omega_{v, s}, \operatorname{ch}_{O_{v}^{n}}\right)=c_{v} Z_{v}(s-\kappa)$ where $c_{v}^{-1}=\lim _{s_{1}, \ldots, s_{r} \rightarrow \infty} Z_{v}(s)$.

Proof. We write $|f(x)|_{v}^{s}=\omega_{v, s}(f(x))=\left|f_{1}(x)\right|_{v}^{s_{1}} \cdots\left|f_{r}(x)\right|_{v}^{s_{r}}$. Then

$$
Z_{v}\left(\omega_{v, s}, \operatorname{ch}_{O_{v}^{n}}\right)=\int_{Y_{v} \cap O_{v}^{n}}|f(x)|_{v}^{s} d_{Y_{v}}(x)
$$

where $d_{Y_{v}}$ is a $G_{v}$-invariant measure with $\int_{Y_{v}^{0}} d_{Y_{v}}=1$. Here $Y_{v}^{0}=\{x \in$ $\left.O_{v}^{n} ;|F(x)|_{v}=1\right\}$ with $F(x)=f_{1}(x) \cdots f_{r}(x)$. Therefore if we put $d_{Y_{v}}(x)=$ 
$c_{v} \frac{d x}{|f(x)|_{v}^{k}}$ with $\int_{O_{v}^{n}} d x=1$, we have

$$
Z_{v}\left(\omega_{v, s}, \operatorname{ch}_{O_{v}^{n}}\right)=c_{v} \int_{Y_{v} \cap O_{v}^{n}}|f(x)|_{v}^{s-\kappa} d x=c_{v} Z_{v}(s-\kappa)
$$

The last equality holds when $\operatorname{Re}(s-\kappa)>0$, i.e., $|0|_{v}^{s-\kappa}=0$, and then it holds for all $s$ by analytic continuation. We have

$$
\begin{aligned}
1 & =\int_{Y_{v}^{0}} d_{Y_{v}}=c_{v} \int_{Y_{v}^{0}} d x=c_{v} \int_{|f(x)|_{v}=1} d x \\
& =c_{v} \lim _{s \rightarrow \infty} \int_{O_{v}^{n}}|f(x)|_{v}^{s} d x=c_{v} \lim _{s \rightarrow \infty} Z_{v}(s)
\end{aligned}
$$

since $f_{i}(x) \in \pi O_{v}$ implies $\lim _{s_{i} \rightarrow \infty}\left|f_{i}(x)\right|_{v}^{s_{i}}=0$.

Lemma 3.2. For a finite place $v$ of $k$,

$$
Z_{v}^{*}\left(\hat{\omega}_{v, s}, \hat{c h}_{O_{v}^{n}}\right)=N\left(d_{v}\right)^{-(d, s)+\frac{n}{2}} \cdot c_{v} Z_{v}^{*}(-s)
$$

where $d_{v}=$ the different of $k_{v}$, and $(d, s)=s_{1} \operatorname{deg} f_{1}+\cdots+s_{r} \operatorname{deg} f_{r}$ where $Z_{v}^{*}(s)$ is the Igusa local zeta function $Z_{v}(s)$ for $f^{*}$.

Proof. By using the self-dual measure, we have $\operatorname{ch}_{O_{v}^{n}}=N\left(d_{v}\right)^{-\frac{n}{2}} \operatorname{ch}_{\left(d_{v}^{-1}\right)^{n}}$ and hence

$$
\begin{aligned}
Z_{v}^{*}\left(\hat{\omega}_{v, s}, \hat{c h}_{O_{v}^{n}}\right) & =Z_{v}^{*}\left(\omega_{v, \kappa-s}, N\left(d_{v}\right)^{-\frac{n}{2}} \operatorname{ch}_{\left(d_{v}^{-1}\right)^{n}}\right) \\
& =N\left(d_{v}\right)^{-\frac{n}{2}} \cdot c_{v} \int_{Y_{v}^{*} \cap\left(d_{v}^{-1}\right)^{n}}\left|f^{*}(x)\right|_{v}^{-s} d x .
\end{aligned}
$$

Now if $d_{v}^{-1}=\pi_{v}^{-t} O_{v}$, then $N\left(d_{v}\right)=q_{v}^{t}$. By the change of variables $x=\pi_{v}^{-t} y$, we have $d x=N\left(d_{v}\right)^{n} d y$ and $\left|f^{*}(x)\right|_{v}^{-s}=N\left(d_{v}\right)^{-(d, s)} \cdot\left|f^{*}(y)\right|_{v}^{-s}$. Hence we obtain our result.

By these lemmas, we obtain the following theorem.

Theorem 3.3. For any finite place $v$ of $k$, we have

$$
Z_{v}\left(\omega_{v, s}, \Phi_{v}\right)=\Gamma_{v}\left(\omega_{v, s}\right) Z_{v}^{*}\left(\omega_{v, \kappa-s}, \hat{\Phi}_{v}\right)
$$

for any $\Phi_{v} \in \mathfrak{S}\left(V_{v}\right)$ where $\Gamma_{v}$ is given by $\Gamma_{v}\left(\omega_{v, s}\right)=N\left(d_{v}\right)^{(d, s)-\frac{n}{2}}$. $Z_{v}(s-\kappa) / Z_{v}^{*}(-s)$ where $Z_{v}(s)=\int_{O_{v}^{n}}|f(x)|_{v}^{s} d x$ and $Z_{v}^{*}(s)=\int_{O_{v}^{n}}\left|f^{*}(x)\right|_{v}^{s} d x$ with $\int_{O_{v}^{n}} d x=1$ is the Igusa local zeta function and $(d, s)=s_{1} \operatorname{deg} f_{1}+\cdots+$ $s_{r} \operatorname{deg} f_{r}$.

\section{Arithmetic calculus (example)}

Theorem 4.1 (Principle of Calculus of Fourier transforms). Let $(G, \rho, V)$ be a reductive regular $P$. $V$. defined over an algebraic number field $k$ satisfying $Z_{a}=$ 
$\tau Z_{m}$. Then for any $\Phi_{\infty} \in \mathfrak{S}\left(V_{\infty}\right)$, we have

$$
\begin{aligned}
Z_{\infty}(\mid & \left.||_{\infty}^{s}, \Phi_{\infty}\right) \cdot \prod_{v \in \Sigma_{f}} c_{v} Z_{v}(s-\kappa) \\
= & \left|D_{k}\right|^{-(d, s)+\frac{n}{2}} \cdot Z_{\infty}^{*}\left(||_{\infty}^{\kappa-s}, \hat{\boldsymbol{\Phi}}_{\infty}\right) \cdot \prod_{v \in \Sigma_{f}} c_{v} Z_{v}^{*}(-s)
\end{aligned}
$$

where $D_{k}$ is the discriminant of $k, Z_{v}(s)=$ the Igusa local zeta function, and $\Sigma_{f}=$ the set of finite places of $k$.

Proof. By Theorem 2.4, Lemmas 3.1 and 3.2 for $\Phi=\Phi_{\infty} \otimes\left(\bigotimes_{v \in \Sigma_{f}} \operatorname{ch}_{O_{v}^{n}}\right)$ and $\omega=||_{\infty}^{s} \cdot \prod_{v \in \Sigma_{f}} \omega_{v, s}$, we obtain our result.

This theorem shows that we can obtain Fourier transforms over $\mathbb{R}$ or $\mathbb{C}$ if we have the explicit form of the Igusa local zeta function. In this section, we shall calculate the Fourier transform of the complex power of the relative invariants of the following $(\tilde{G}, \rho, V)$ where $V=\left\{\tilde{x}=(X ; y, z, w) ;{ }^{t} X=\right.$ $\left.-X \in M_{2 m+1}, y, z, w \in M_{2 m+1,1}\right\}$, and $\rho(g) \tilde{x}=\left(\alpha A X^{t} A ; \beta A y, \gamma^{t} A^{-1} z\right.$, $\left.\delta^{t} A^{-1} w\right)$ for $g=(\alpha, \beta, \gamma, \delta ; A) \in \tilde{G}=G L_{1}^{4} \times S L_{2 m+1}$ with $m \geq 2$. Then $\operatorname{ker} \rho=\left\{1,\left(1,-1,-1,-1 ;-I_{2 m+1}\right)\right\}$. Put

$$
\xi=\left(\left(\begin{array}{cc|c}
0 & I_{m} & 0 \\
-I_{m} & 0 & 0 \\
\hline 0 & 0
\end{array}\right) ; e_{2 m+1}, e_{1}+e_{2 m+1}, e_{m+1}+e_{2 m+1}\right)
$$

where

$$
e_{i}={ }^{t}(0, \ldots, 0, \stackrel{i}{1}, 0, \ldots, 0) \text {. }
$$

Then

$$
f_{1}(\tilde{x})=\text { Pfaffian of }\left(\begin{array}{c|c}
X & y \\
--^{t} y & 0
\end{array}\right)
$$

is a relative invariant corresponding to $\chi_{1}(g)=\alpha^{m} \beta$. Similarly, $f_{2}(\tilde{x})=$ $\langle y, z\rangle$ (resp. $\left.f_{3}(\tilde{x})=\langle y, w\rangle, f_{4}(\tilde{x})={ }^{t} z X w\right)$ is a relative invariant corresponding to $\chi_{2}(g)=\beta \gamma$ (resp. $\left.\chi_{3}(g)=\beta \delta, \chi_{4}(g)=\alpha \gamma \delta\right)$. Then we have $S=\left\{f_{1}=0\right\} \cup\left\{f_{2}=0\right\} \cup\left\{f_{3}=0\right\} \cup\left\{f_{4}=0\right\}$ with $V-S=\rho(G) \cdot \xi$ and a generic isotropy subgroup $\rho\left(G_{\xi}\right)=S p_{m-1}$ is connected semisimple. We have $\operatorname{det} \rho(g)^{2}=\chi_{1}^{2(2 m-1)} \chi_{2}^{2} \chi_{3}^{2} \chi_{4}^{4 m}$, i.e., $\kappa=(2 m-1,1,1,2 m)$. By [K-K], this P.V. satisfies $Z_{a}=\tau Z_{m}$ for some $\tau>0$.

Proposition 4.2 (Hosokawa). Let $K$ be a p-adic field with the maximal compact subring $O_{K}$. Let $d x$ be the Haar measure on $V_{K}$ satisfying $\int_{V\left(O_{K}\right)} d x=1$. Then the Igusa local zeta function $Z_{K}(s)=\int_{V\left(O_{K}\right)}\left|f_{1}(x)\right|_{K}^{s_{1}} \cdots\left|f_{4}(x)\right|_{K}^{s_{4}} d x$ is given by

$$
\begin{aligned}
Z_{K}(s)= & \prod_{i=1}^{m} \frac{(2 i-1)}{1-q^{-\left(s_{1}+2 i-1\right)}} \cdot \prod_{k=2}^{4} \frac{(1)}{1-q^{-\left(s_{k}+1\right)}} \\
& \cdot \frac{(2 m)}{1-q^{-\left(s_{4}+2 m\right)}} \cdot \frac{(2 m+1)}{1-q^{-\left(s_{s}+2 m+1\right)}}
\end{aligned}
$$


where $(i)=1-q^{-i}, s_{5}=s_{1}+\cdots+s_{4}$ and $q=$ the module of $K$.

Now we take $k=\mathbb{Q}$ in Theorem 4.1 and we have

$$
\int_{V_{\mathbf{R}}-S_{\mathbf{R}}}|f(x)|_{\mathbb{R}}^{s} \hat{\boldsymbol{\Phi}}_{\mathbb{R}}(x) d x=\gamma(s) \int_{V_{\mathbf{R}}-S_{\mathbf{R}}}|f(x)|_{\mathbb{R}}^{-s-\kappa} \Phi_{\mathbb{R}}(x) d x
$$

with $\gamma(s)=\prod_{p} c_{p} Z_{p}(-s-\kappa) / \prod_{p} c_{p} Z_{p}(s)$ since

$$
Z_{\mathbb{R}}\left(||_{\mathbb{R}}^{s}, \Phi_{\mathbb{R}}\right)=\int_{V_{\mathbb{R}}-S_{\mathbb{R}}}|f(x)|_{\mathbb{R}}^{s-\kappa} \Phi_{\mathbb{R}}(x) d x .
$$

Since

$$
c_{p}^{-1}=\lim _{s_{1}, \ldots, s_{4} \rightarrow \infty} Z_{\mathbb{Q}_{p}}(s)=\prod_{i=1}^{m}(2 i-1) \cdot(1)^{3} \cdot(2 m) \cdot(2 m+1)
$$

by Lemma 3.1 and Proposition 4.2, we have

$$
\prod_{p} c_{p} Z_{p}(s)=\prod_{i=1}^{m} \zeta\left(s_{1}+2 i-1\right) \cdot \prod_{k=2}^{4} \zeta\left(s_{k}+1\right) \cdot \zeta\left(s_{4}+2 m\right) \cdot \zeta\left(s_{5}+2 m+1\right)
$$

where $\zeta(s)$ is the Riemann zeta function. Since $-s-\kappa=\left(-s_{1}-2 m+1,-s_{2}-\right.$ $\left.1,-s_{3}-1,-s_{4}-2 m\right)$, we have

$$
\begin{aligned}
\prod_{p} c_{p} Z_{p}(-s-\kappa)= & \prod_{i=1}^{m} \zeta\left(-s_{1}-2 i+2\right) \cdot \prod_{k=2}^{3} \zeta\left(-s_{k}\right) \cdot \zeta\left(-s_{4}+1-2 m\right) \\
& \cdot \zeta\left(-s_{4}\right) \cdot \zeta\left(-s_{5}-2 m\right) .
\end{aligned}
$$

Thus, by using the functional equation of the Riemann zeta function:

$$
\zeta(-s) / \zeta(1+s)=(2 \pi)^{-s-1} \cdot 2 \cdot\left(-\sin \frac{\pi s}{2}\right) \cdot \Gamma(1+s),
$$

we have

$$
\begin{aligned}
\gamma(s)=\prod_{p} c_{p} Z_{p}(-s-\kappa) / \prod_{p} c_{p} Z_{p}(s) \\
=\prod_{i=1}^{m} \frac{\zeta\left(-\left(s_{1}+2 i-2\right)\right)}{\zeta\left(1+\left(s_{1}+2 i-2\right)\right)} \cdot \prod_{k=2}^{4} \frac{\zeta\left(-s_{k}\right)}{\zeta\left(1+s_{k}\right)} \cdot \frac{\zeta\left(-\left(s_{4}+2 m-1\right)\right)}{\zeta\left(1+\left(s_{4}+2 m-1\right)\right)} \cdot \frac{\zeta\left(-\left(s_{5}+2 m\right)\right)}{\zeta\left(1+\left(s_{5}+2 m\right)\right)} \\
=\prod_{i=1}^{m}(2 \pi)^{-s_{1}-2 i+1} \cdot 2 \cdot\left(-\sin \frac{\pi\left(s_{1}+2 i-2\right)}{2}\right) \cdot \Gamma\left(s_{1}+2 i-1\right) \\
\cdot \prod_{k=2}^{4}(2 \pi)^{-s_{k}-1} \cdot 2 \cdot\left(-\sin \frac{\pi s_{k}}{2}\right) \cdot \Gamma\left(1+s_{k}\right) \\
\cdot(2 \pi)^{-s_{4}-2 m} \cdot 2 \cdot\left(-\sin \frac{\pi\left(s_{4}+2 m-1\right)}{2}\right) \cdot \Gamma\left(s_{4}+2 m\right) \\
\cdot(2 \pi)^{-s_{5}-2 m-1} \cdot 2 \cdot\left(-\sin \frac{\pi\left(s_{5}+2 m\right)}{2}\right) \cdot \Gamma\left(s_{5}+2 m+1\right)
\end{aligned}
$$




$$
\begin{aligned}
& =(2 \pi)^{-t} \cdot(-2)^{m+5} \cdot \prod_{i=1}^{m} \sin \frac{\pi\left(s_{1}+2 i-2\right)}{2} \cdot \prod_{k=2}^{4} \sin \frac{\pi s_{k}}{2} \cdot \sin \frac{\pi\left(s_{4}+2 m-1\right)}{2} \\
& \cdot \sin \frac{\pi}{2}\left(s_{1}+s_{2}+s_{3}+s_{4}+2 m\right) \cdot \prod_{i=1}^{m} \Gamma\left(s_{1}+2 i-1\right) \cdot \prod_{k=2}^{4} \Gamma\left(s_{k}+1\right) \\
& \cdot \Gamma\left(s_{4}+2 m\right) \cdot \Gamma\left(s_{1}+s_{2}+s_{3}+s_{4}+2 m+1\right) \\
& \quad \text { with } t=(m+1) s_{1}+2 s_{2}+2 s_{3}+3 s_{4}+(m+2)^{2} .
\end{aligned}
$$

\section{REFERENCES}

[Gyoja] A. Gyoja, Functional equations for Igusa local zeta functions, in preparation.

[Hosokawa] H. Hosokawa, Some results on Igusa local zeta functions associated with simple prehomogeneous vector spaces, preprint.

[Igusa 1] J. Igusa, Some results on p-adic complex powers, Amer. J. Math. 106 (1984), 10131032.

[Igusa 2] _ On functional equations of complex powers, Invent. Math. 85 (1986), 1-29.

[Igusa 3] _ On a certain class of prehomogeneous vector spaces, J. Pure Appl. Algebra 47 (1987), 265-282.

[Igusa 4] Zeta distributions associated with some invariants, Amer. J. Math 109 (1987), $1-34$.

[Iwasawa] K. Iwasawa, A note on L-functions, Proc. Internat. Congress Math. (Cambridge, Mass., 1950), vol. 1, Amer. Math. Soc., Providence, RI, 1952, p. 322; A letter to J. Dieudonné (1952), Appendix in Adv. Stud. Pure Math. 21 (1992).

[K-K] T. Kimura and T. Kogiso, On adelic zeta functions of prehomogeneous vector spaces with a finitely many adelic open orbits, Adv. Stud. Pure Math. 21 (1992), 21-31.

[K-K-H] T. Kimura, S. Kasai, and H. Hosokawa, Universal transitivity of simple and 2-simple prehomogeneous vector spaces, Ann. Inst. Fourier (Grenoble) 38 (1988), 11-41.

[K-K-M] M. Kashiwara, T. Kimura, and M. Muro, Microlocal calculus of Fourier transforms over $\mathbb{R}$, in preparation.

[Kimura] T. Kimura, The b-functions and holonomy diagrams of irreducible regular prehomogeneous vector spaces, Nagoya Math. J. 85 (1982), 1-80.

[M] M. Muro, Microlocal analysis and calculations on some relatively invariant hyperfunctions related to zeta functions associated with the vector spaces of quadratic forms, Publ. Res. Inst. Math. Sci. 22 (1986), 395-463.

[Ono] T. Ono, An integral attached to a hypersurface, Amer. J. Math. 90 (1968), 1224-1236.

[F. Sato 1] F. Sato, Zeta functions in several variables associated with prehomogeneous vector spaces. II : a convergence criterion, Tôhoku Math. J. 35 (1983), 77-99.

[F. Sato 2] Z Z Z Z L functions in several variables associated with prehomogeneous vector spaces. I, III , Tôhoku Math. J. 34 (1982), 437-483; Ann. of Math. (2) 116 (1982), 117-212

[S-K] M. Sato and T. Kimura, A classification of irreducible prehomogeneous vector spaces and their relative invariants, Nagoya Math. J. 65 (1977), 1-155.

[S-S] M. Sato and T. Shintani, On zeta functions associated with prehomogeneous vector spaces, Ann. of Math. (2) 100 (1974), 131-170.

[Tate] J. Tate, Fourier analysis in number fields and Hecke's zeta functions, Ph.D. thesis, Princeton, 1950; Algebraic Number Theory (Cassels and Fröhlich, eds.), Thompson, Washington, D.C., 1967, pp. 305-347.

The Institute of Mathematics, University of Tsukuba, Ibaraki, 305, Japan

E-mail address: kimurata@math.tsukuba.ac.jp 